\title{
LAS IDENTIDADES DE GÉNERO SEGÚN LAS Y LOS ADOLESCENTES. PERCEPCIONES, DESIGUALDADES Y NECESIDADES EDUCATIVAS
}

\author{
Guadalupe Calvo García \\ Universidad de Cádiz
}

\begin{abstract}
RESUMEN: Este artículo recoge algunos de los hallazgos de una tesis doctoral cuyos fundamentos teóricos defienden que las identidades son construcciones simbólicas surgidas de las categorías que cada sociedad establece, y parte del planteamiento de que nuestra sociedad occidental únicamente legitima las identidades de género "hombre" y "mujer", que presenta como naturales. En este sentido, señalamos que esta categorización binaria provoca desigualdades entre las personas; tanto entre quienes se incluyen en ellas, como, especialmente, entre quienes no encajan en el binomio. Desde esta perspectiva desarrollamos una investigación cualitativa basada en la información proporcionada por adolescentes formados como mediadoras y mediadores en salud y sexualidad a través del programa educativo Forma Joven de la Junta de Andalucía, la cual se materializa en cuatro estudios de caso. Concretamente, en nuestro trabajo mostramos cómo entienden nuestros sujetos de estudio que han construido sus identidades sexuales y han llegado a convertirse en las personas que son, además de analizar la valoración que hacen, tanto de esas identidades propias como de las que asumen otros chicos y chicas de su edad. Como conclusión, aportamos una reflexión sobre las necesidades educativas en relación al género que se extraen de los discursos de estos y estas adolescentes.
\end{abstract}

PALABRAS CLAVE: Identidades de género, adolescencia, desigualdades, necesidades educativas.

\section{GENDER IDENTITIES ACCORDING TO THE ADOLESCENTS. PERCEPTIONS, INEQUALITIES AND EDUCATIONAL NEEDS}

ABSTRACT: This article gathers some of the findings from a doctoral thesis whose theoretical foundations defend that identities are symbolic constructions arising from the categories that each society establishes, and is based on the proposition that our western society just legitimizes the gender identities 
"man" and "woman", presenting them as natural. In this way, we point out that this binary categorization causes inequalities between persons; both among those included in them, and, especially, among those who don't fit into the binomial. From this perspective, we develop a qualitative research based on the information provided by adolescents trained as mediators in health and sexuality by the educational program Forma Joven of the Regional Government of Andalusia, which is materialized in four case studies. Specifically, in our work we show how our subjects of study understand that they have built their sexual identities and have become the persons who they are, besides analyzing the assessment they make, both those identities of their own and those assumed by other children and girls of her age. In conclusion, we provide a reflection on the educational needs related to gender that are extracted from the discourses of these adolescents.

KEYWORDS: Gender identities, adolescence, inequalities, educational needs.

Recibido: 11/04/2017

Aceptado: 28/09/2017

Correspondencia: Guadalupe Calvo García, Universidad de Cádiz, Facultad de Ciencias de la Educación, Campus de Puerto Real, 11519 Cádiz. Email: guadalupe.calvo@uca.es.

\section{INTRODUCCIÓN}

En nuestro artículo presentamos algunos de los hallazgos de la tesis doctoral titulada "La construcción de las identidades sexuales en la adolescencia. Cuatro estudios de caso de mediadoras y mediadores del programa educativo Forma Joven", la cual se desarrolla desde un planteamiento teórico que entiende que las identidades de género no son el simple resultado de un proceso complejo de socialización, sino verdaderas construcciones simbólicas surgidas de las categorías que cada sociedad establece y en función de las cuales se organiza. En consecuencia, en este documento cuestionamos el binarismo de género que sustenta nuestra sociedad occidental, el cual "obliga" a las personas a posicionarse como hombres o como mujeres, y señalamos las desigualdades a las que este da lugar. Esta tarea la hacemos apoyándonos en el análisis de los casos de dos chicos y dos chicas adolescentes que se han formado como mediadoras en salud y sexualidad a través del programa educativo Forma Joven de la Junta de Andalucía.

Concretamente, en el presente trabajo mostramos cómo entienden nuestros sujetos de estudio que han construido sus identidades de género y han Ilegado a convertirse en las personas que son, además de analizar las experiencias de desigualdad vinculadas a las mismas que detectan, y la valoración que hacen tanto de dichas identidades propias como de las que asumen otros chicos y chicas de su edad. A modo de conclusión, aportamos una reflexión sobre las necesidades educativas en relación al género que se extraen de los discursos de estos y estas adolescentes. 


\section{UNA SOCIEDAD SUSTENTADA EN EL BINARISMO DE GÉNERO}

Si hay una premisa básica que sustenta nuestra sociedad occidental, esta se refiere a que los humanos nos dividimos en hombres y mujeres. Esta concepción binarista es cuestionada exclusivamente desde ciertas perspectivas de los estudios de género y desde corrientes del activismo social LGTBI ${ }^{1}$, afines a lo Queer (Vidarte, Sáez y Córdoba, 2007), cuyos discursos se han introducido muy tímidamente en las instituciones públicas de países como Alemania o Australia, que ofrecen la posibilidad de no registrarse con género femenino o masculino; medida que podría contribuir a la estigmatización de quienes seleccionaran dicha opción si su puesta en marcha no fuera acompañada de un intensa labor educativa acerca de la diversidad sexual (Alonso, 2013).

En este sentido, podemos afirmar que la sociedad occidental ha naturalizado las identidades de género binarias y heterosexuales, las cuales son asignadas en función de los genitales y reducen a dos grupos la interminable diversidad humana. El binomio de género que estructura la sociedad occidental contemporánea obvia que un $1 \%$ de los bebés que nacen en el mundo experimentan lo que se conoce como intersexualidad; es decir, presentan un desajuste (según lo esperado) entre el sexo genético, las gónadas y los genitales (Audí, 2016, citado por Ayuso, 2016). En relación a este hecho Anne Fausto-Sterling desarrolla su teoría en la que defiende la existencia de, al menos, cinco sexos claramente identificables (Fausto-Sterling, 1998).

Igualmente, el binarismo de género prevalente patologiza la transexualidad. Muestra de ello es que los "trastornos de la identidad de género" se han mantenido en el principal manual de diagnóstico de trastornos mentales, el DSM hasta su cuarta versión, sustituida en 2014 por el DSM V, donde se continúa manteniendo como categoría diagnóstica la "disforia de género". Coherentemente con esta perspectiva patologizadora, la principal opción que se ofrece a estas personas para su integración en la sociedad binaria es la cirugía de reasignación sexual y el cambio de la mención relativa al sexo en su documento nacional de identidad.

Sin embargo, van surgiendo voces desde estas posiciones que reivindican el reconocimiento de la diversidad humana optando por el "transgenerismo", es decir, por no definirse según uno de los dos género legitimados; opción respaldada por las leyes contra la discriminación sexual recientemente aprobadas en España, iniciadas con la Ley 2/2014 de 8 de julio, integral para la no discriminación por motivos de identidad de género y reconocimiento de los derechos de las personas transexuales de Andalucía.

Con respecto a la homosexualidad, en ella se da la relación esperada entre el sexo y el género, y es la orientación de los deseos sexuales la que se enfrenta a la heterosexualidad dominante; en la actualidad se suple la "deficiencia" que excluye a quienes se identifican como homosexuales, orientándoles al desarrollo de los patrones heteronormativos que tradicionalmente han asumido quienes encajan en el binomio de género; de manera que, siguiendo a Guasch (2007), los homosexuales se han convertido en heterosexuales.

1. Lesbiano, gay, transexual, bisexual e intersexual. 


\section{LA CONSTRUCCIÓN PERFORMATIVA DE LAS IDENTIDADES DE GÉNERO}

Como acabamos de evidenciar, y en concordancia con el "sistema sexo-género" propuesto por Gayle Rubin (1996), las identidades de género que cuentan con legitimidad en la actual sociedad occidental no se corresponden en absoluto con la diversidad humana, sino con las necesidades de esta sociedad para mantenerse y reproducirse. Es por esto que, principalmente en el ámbito de la antropología, numerosos estudios nos muestran que en contextos sociales no tan fagocitados por la cultura occidental a causa de la globalización, se reconocen identidades de género distintas a las nuestras.

Buena cuenta de ello da Bolin (1996) en su clasificación de cinco modelos de varianza del género detectadas a nivel global, e igualmente, deja constancia del carácter construido de las identidades de género, Francisco Vázquez $(2007,2009)$, quien propone tres regímenes de verdad en relación a estas a lo largo de la historia.

El que las categorías identitarias difieran de unas sociedades a otras, tanto en el eje espacial como en el temporal, muestra que dichas identidades no son naturales ni universales, sino que se construyen socialmente. La perspectiva teórica que a nuestro entender mejor explica dicho proceso de construcción es la de la performatividad desarrollada por Judith Butler $(2007,2008)$.

Butler explica que construimos nuestra identidad a través de una performance que realizamos de manera continua, inspirada en los modelos que nuestra sociedad nos propone para la categoría de género en la que nos ha posicionado. Por tanto, aunque cada persona posea una actitud activa en este proceso siempre inacabado, no decidimos libremente el papel que vamos a representar. Esto significa que es la sociedad la que propone los modelos identitarios que necesita a través de los discursos que difunde desde sus estructuras de poder, y de ese modo consigue que dichas identidades sean asumidas por la mayoría de los sujetos que forman parte de la misma.

\section{LOS RIESGOS DE NO ASUMIR UNA IDENTIDAD NORMATIVA EN LA INFANCIA Y LA ADOLESCENCIA}

El principal motivo por el que reflexionamos acerca de la construcción de las identidades de género y nos esforzamos por deconstruir la concepción binaria de las mismas, lo encontramos en las desigualdades que dicha categorización genera entre las personas. En primer lugar, podemos señalar las importantes desigualdades existentes entre quienes asumen las identidades normativas (mujeres / hombres), pero especialmente debemos destacar las que experimentan quienes quedan excluidos del binomio heterosexual.

Las desigualdades a las que nos referimos comienzan en la infancia y se radicalizan en la adolescencia. La escuela, que según Pérez Gómez (1992) tiene la función de socializar y la de educar, respecto a la cuestión que nos ocupa parece limitarse a socializar según las normas de género vigentes sin educar para la diversidad sexual.

Especialmente relevantes nos resultan los estudios de Emma Renold, en los que revela cómo las chicas tienden a posicionarse cercanas al modelo de feminidad hegemónico, caracterizado por los estereotipos sobre la sexualidad femenina (2000, 
2001b) y cómo los chicos para no ser marginados deben adecuarse al modelo de masculinidad dominante, en el cual es fundamental la heterosexualidad (2000, 2001a, 2003, 2004).

Respecto a la diversidad sexual, Generelo y Pichardo (2006) señalan que la homofobia está presente en la gran mayoría de los centros educativos, siendo silenciada por la comunidad. Sánchez Sainz (2014), que focaliza la atención en el alumnado de Educación Infantil y Primaria, indica que el rechazo comienza en los últimos años de la primaria, y que puede detectarse especialmente a través de los insultos que se dirigen a quienes no asumen identidades normativas y de la escasa valoración con la que cuentan las familias no tradicionales.

En cuanto al alumnado adolescente, Pichardo (2009) destaca su desconocimiento generalizado de las personas que no se identifican como heterosexuales $y$, en consecuencia, su dificultad para construir identidades no normativas sanas y felices. En esta línea, Platero (2008, 2010) se refiere al "bullying homofóbico", el cual se dirige a chicas y chicos que no representan los modelos de género normativos independientemente de si mantienen relaciones homosexuales o no.

A tenor del panorama dibujado, Pichardo (2009) cuestiona el tabú existente en nuestra sociedad en relación a la educación para la diversidad sexual, subrayando que el $16 \%$ de las y los adolescentes entrevistados afirmaban no sentirse heterosexuales. Pero la escasa oferta educativa en este ámbito en las escuelas es comprensible si tenemos en cuenta que el propio profesorado universitario que debería proporcionar formación a los maestros y las maestras, carece de ella (Penna, 2015), lo que contribuye a que el $15 \%$ de los futuro docentes de secundaria presenten incluso actitudes homófobas (Penna y Sánchez, 2015).

\section{Nuestra inVEStigaCión SObre las IDENTIDADES DE GÉNERO en la ADOLESCENCIA}

En nuestra tesis doctoral quisimos focalizar la atención en la adolescencia, por valorarla como una etapa de la vida especialmente interesante en lo que se refiere a los procesos de identificación. Esta decisión nos permitiría escuchar a chicas y chicos hablar en primera persona acerca de sus experiencias, inquietudes, conflictos, dificultades, expectativas, ilusiones... y analizar su propio discurso.

Se convirtieron en nuestros sujetos de estudio los y las adolescentes que se formaron como mediadores de salud a través del programa educativo de la Junta de Andalucía "Forma Joven" ${ }^{2}$ entre noviembre de 2010 y abril de 2013. Consideramos que este grupo de adolescentes, que ya había comenzado a reflexionar sobre las desigualdades, podría enriquecernos y ayudarnos especialmente a comprender el objeto de estudio.

El objetivo general de nuestra investigación se orientaba al conocimiento de las percepciones, creencias e interpretaciones de las y los adolescentes acerca de las identidades sexuales, tanto propias como de sus iguales, y de las desigualdades relacionadas con dicho concepto.

2. Ver: www.formajoven.org. 
Este objetivo general se concretó en cinco objetivos específicos o dimensiones de estudio en las que nos interesaba indagar, ocupándonos en este artículo de las dos primeras:

1. Conocer las opiniones de las y los adolescentes acerca de cómo les han influido los diferentes agentes educativos, tanto en la construcción de su propia identidad sexual, como en la comprensión de otros modelos de identidad asumidos por sus iguales.

2. Descubrir de qué modo valoran las y los adolescentes los distintos modelos de identidades sexuales y a sus iguales que asumen identidades no normativas.

A pesar de que nuestro estudio prestaba atención a todos los chicos y las chicas participantes en "Forma Joven", nos interesaba investigar las particularidades de casos distintivos (Simons, 2011, p. 20), por lo que este derivó en los estudios de los casos de cuatro de dichos participantes cuyas historias resultaban relevantes para el conjunto de la investigación.

Para la recogida de información, recurrimos a las principales técnicas e instrumentos propios de la investigación cualitativa: la observación etnográfica participante, las entrevistas en profundidad y las conversaciones informales.

La observación participante constituyó la principal estrategia de recogida de información utilizada en las sesiones formativas, debido a que tuvimos la posibilidad de integrarnos completamente en el contexto objeto de estudio y acompañar a los diferentes grupos a lo largo de sus procesos formativos. Concretamente, observamos en 5 cursos de formación desarrollados durante fines de semanas completos y en 2 jornadas formativas de un día.

En la primera formación en la que participamos, seleccionamos a los y las protagonistas de los casos ayudándonos de un cuestionario, teniendo como criterios: el interés por participar y la diversidad sexual; con el objetivo de que sus historias nos proporcionaran distintas visiones, incluso complementarias, de la temática que nos ocupa. Elegimos el mismo número de chicas que de chicos pero con opciones sexuales diversas, finalmente fueron: Fernando, Alicia, David y Elena ${ }^{3}$.

La principal técnica utilizada para el desarrollo del trabajo fue la entrevista en profundidad, que permitió que entre nosotros se generara la confianza suficiente para poder conversar sobre sus experiencias y concepciones en relación al sexo, al género y a la sexualidad. Con Fernando realizamos cuatro: dos en 2011, una en 2012 y otra en 2013. Con el resto llevamos a cabo tres: dos con cada uno en 2011 y otra a finales de 2012 o principios de 2013.

Respecto a las conversaciones informales, estas resultaron de gran valor, debido a que al no estar programadas, sino surgir espontáneamente, en ellas suelen fluir las verdaderas concepciones que se han interiorizado. Han tenido lugar desde la primera sesión de "Forma Joven" hasta el fin de las entrevistas, no solo de manera presencial, también por teléfono y correo electrónico.

El proceso de análisis de la información recopilada a través de las diferentes técnicas expuestas coincidió en gran medida con modelo "de transformación de datos" propuesto por Wolcott (1994), el cual distingue tres momentos de organización e interpretación de estos: la descripción de los mismos, su análisis y su interpretación.

3. Los nombres son ficticios para garantizar el anonimato de los sujetos de estudio. 
La descripción de los datos comenzó con la transcripción de las entrevistas. Una vez realizado este trabajo y localizadas en el diario de campo todas las reflexiones surgidas de las observaciones y de las conversaciones informales, entramos de lleno en la codificación y la organización de los mismos. En primer lugar, distinguimos en cada uno de los casos los diferentes ámbitos que fueron emergiendo del discurso de los y las adolescentes, y fuimos identificándolos y marcándolos en las transcripciones de las entrevistas y demás material. A continuación, diseñamos mapas conceptuales que dotaran de estructura a los distintos ámbitos temáticos considerados, los cuales constituirían las plantillas a partir de las cuales se desarrollarían los informes de cada uno de los casos.

Para el análisis y la interpretación de la información, se recurrió a las dimensiones de estudio y, a través de la puesta en relación de los datos recogidos en cada uno de los informes con las teorías que fundamentaban la investigación, se fue desarrollando el análisis de las percepciones, creencias, opiniones, experiencias... de las y los sujetos de estudio relativas a cada una de ellas.

\section{Lo QUe NOS enSEÑAN NUESTROS CASOS ACERCA DE LAS IDENTIDADES DE GÉNERO}

La primera enseñanza que nos aportan nuestros sujetos de estudio surge de sus propios posicionamientos en relación a las normas de género, que en ningún caso coinciden con los modelos hegemónicos de feminidad o masculinidad.

A Fernando, el hecho de mantener relaciones homosexuales le aparta del arquetipo viril (Moreno, 1988) dominante en nuestra sociedad, en el cual es fundamental la heterosexualidad; y quizás la marginalidad que implica posicionarse como homosexual, puesta de relieve en varios de los estudios expuestos previamente, contribuyó a que no lo hiciera hasta los 17 años cuando estableció una relación amorosa estable con otro chico. No obstante, lo que explicita en su discurso es que su resistencia a definirse de ese modo era debida principalmente a la no identificación con los modelos homosexuales más difundidos; algo que resulta paradójico teniendo en cuenta, por ejemplo, el papel central del baile en su vida, actividad tradicionalmente asociada a lo femenino y a los hombres homosexuales.

En cuanto a Alicia, aunque hasta sus 17 años sólo ha mantenido relaciones amorosas y sexuales con chicos, por lo que se considera heterosexual, también transgrede en algunos aspectos los modelos femeninos hegemónicos. En primer lugar, en ningún momento asume una identidad subordinada; es una chica con una gran autoestima, con ganas de experimentar en los diversos ámbitos de su vida, que quiere irse lejos de su pueblo para poder ser más independiente y que, incluso, se plantea la posibilidad de mantener relaciones homosexuales. Por otra parte, Alicia reproduce algunos roles tradicionalmente masculinos como la práctica cuasi profesional del baloncesto, el consumo de alcohol y tabaco o el mantenimiento de relaciones sentimentales/sexuales paralelas y no estables; en la línea propuesta por Duque (2006).

David, entre los 14 y los 16 años, podría asemejarse más a los modelos hegemónicos de masculinidad; es un chico asentado en la heterosexualidad que en ningún momento se ha planteado la posibilidad de transgredir la categoría en la que se 
posiciona, pero que, en contra de lo que promueve el arquetipo viril, es tímido y callado, asume responsabilidades domésticas, tiene como principal actividad de ocio "cotillear" y rechaza los hábitos de riesgo típicamente masculinos, como el consumo de alcohol y la violencia.

Elena, de 20 años, directamente se define como lesbiana, asemejándose a lo que en el ámbito de la homosexualidad femenina se denomina modelo butch, contrario a los estereotipos relativos a la sexualidad femenina. No obstante, en concordancia con los planteamientos de Guasch (2007), esta chica sí que asume los modelos de relación tradicionalmente heterosexuales y, por otra parte, las tareas domésticas típicamente femeninas, frente a la irresponsabilidad de las personas con quienes comparte alojamiento.

\subsection{Las influencias que detectan en sus propios procesos de construcción identitaria}

Resulta curioso que, aunque la mayoría de investigaciones educativas revisadas centren su atención en la escuela, ninguno de nuestros sujetos de estudio se refiera a esta como un agente importante en su proceso identitario, y que por unanimidad señalen a sus padres como los principales responsables de cómo son actualmente.

Fernando identifica desde un primer momento a sus padres y a sus amistades como las personas que más han contribuido a su construcción como persona. Respecto a los primeros, indica que él es una mezcla de ambos y que de ellos lo ha aprendido casi todo; además, de su discurso se desprende que ha sido determinante en la construcción de su identidad la actitud respetuosa que ambos siempre han tenido hacia él, permitiéndole "que sea como quiera ser". Sobre sus amistades, con 15 años exponía que le habían hecho madurar por ser mayores que él y por tener una mentalidad abierta en lo relativo al género y a la sexualidad, lo que en su opinión permitía que en su grupo pudieran actuar libremente, sin atenerse a los estereotipos de género ni a las "normas" acerca de la sexualidad, y sin tener que definirse.

Alicia, igualmente, identifica a sus padres como los agentes más influyentes en la construcción de su identidad. Esta chica es capaz de explicar qué características de la educación que sus progenitores le han proporcionado la han llevado a ser tan diferente del resto de sus amistades, considerando clave el que nunca le hayan prohibido nada y que simplemente le hayan pedido que cumpla con sus responsabilidades.

David inicialmente no sabe si ha sido su padre o su madre quien más le ha influido para llegar a ser como es. Por algunos de sus comentarios se deduce que entiende que cuando era más joven aprendía más de su madre, por compartir más tiempo con ella; pero parece que su padre ha contribuido a favorecer su acercamiento al arquetipo viril defendiéndole cuando su madre le regañaba por tener novia, retrasándole la hora de vuelta a casa o dándole dinero para salir.

Elena afirma haber adquirido el carácter duro de su madre y el transgresor de su padre, un hombre profundamente católico que ha mantenido una lucha continua contra las normas de género instauradas en los diferentes contextos en lo que ha tenido que desenvolverse (en el escolar, cursando la asignatura de "Labores del hogar", en el laboral, dirigiendo la revista "El floripondio", etc.). La chica alude a los comentarios que le dirigen su madre y sus tías acerca de sus semejanzas con su 
padre, fundamentalmente en las rarezas. Si bien, es cierto que Elena es parecida a su padre en lo que se refiere a la transgresión de lo normativo, también lo es en su actitud reivindicativa y solidaria.

\subsection{Sus explicaciones acerca de la construcción de las identidades de género}

Aunque los y las protagonistas de los casos identifican a sus padres (Fernando, también a sus amistades) como las personas más influyentes en su desarrollo personal, cuando centramos la atención en el género y la sexualidad surgen las dudas sobre si el contexto influye o estamos determinados biológicamente.

Fernando, que con 15 años pensaba que lo natural era ser heterosexual, con 17 afirmaba que "en esta vida hay tanto heterosexuales como homosexuales", dando a entender que las identidades están determinadas y que las categorías son cerradas y estables; a pesar de que él mismo ha rechazado posicionarse como homosexual a lo largo de su adolescencia. El chico entra en contradicción al ser cuestionado acerca de por qué la mayoría de las personas se definen como heterosexuales, lo que explica aludiendo a la educación y la sociedad, que establece cómo deben ser los niños y cómo, la niñas; y vuelve a contradecirse al recurrir a la genética para explicar la diversidad sexual humana.

Alicia parece tenerlo más claro. Sus explicaciones reflejan que entiende la construcción de las identidades sexuales de manera performativa, ya que considera que es nuestra sociedad, presuponiendo una heterosexualidad natural, la que nos empuja a actuar como heterosexuales. La chica lo ejemplifica aludiendo a la prohibición que se impone a niños y niñas de ducharse juntos. Al hacerse consciente de este hecho, la chica llega a plantearse la posibilidad de mantener relaciones bisexuales.

David es mucho más escueto en su explicación de por qué hay personas que asumen identidades normativas y otras que no, y simplemente expone "que cada uno tiene sus gustos", como en el resto de los aspectos de la vida.

Del discurso de Elena se deduce que piensa que las identidades sexuales son innatas y que los roles de género son producto de un proceso de socialización (que no de construcción, como defiende Judith Butler). Con sus explicaciones acerca de la actitud subordinada que asumen las chicas de su instituto, la cual considera consecuencia de los discursos románticos que les llegan por medio de revistas y series juveniles, la chica se refiriere a un proceso de socialización que modelaría una supuesta realidad prediscursiva, y no a un proceso performativo de construcción de la identidad. Elena es, de los cuatro, la que entiende las identidades sexuales de un modo más determinista, lo confirma la argumentación que ofrece a su madre cuando esta le reprocha que salga con chicas, en la que compara ser homosexual con tener una discapacidad": "(...) eso viene conmigo (...) te puede salir un niño subnormal y a ti te ha salido una hija bollera".

Elena justifica esta concepción acerca de la identidad de género explicando que desde pequeña se ha sentido atraída por otras chicas y nunca por chicos. Al respecto, merece especial mención la anécdota que protagonizó cursando $3^{\circ}$ de Primaria, cuando en mitad de la clase le dijo a su maestra que de mayor cambiaría de sexo

4. Como si estas no dependieran igualmente del contexto social. 
porque creía que le gustaban las mujeres y entonces ella no podía ser otra mujer. Este acontecimiento refleja que, a sus 8 años, ya había interiorizado las normas del

"Régimen de verdad del sexo simulacro" identificado por Vázquez (2009) y necesitaba buscar, a través de la modificación de su cuerpo, la relación esperada en nuestra sociedad, entre el sexo, el género y la orientación sexual.

Elena muestra de nuevo su concepción determinista acerca de la identidad sexual cuando alude a su expareja Alba, a quien, tras la relación que mantuvieron durante dos años, "le dio por la vena hetero de repente"; lo que a ella le desconcierta y explica aludiendo a la juventud de esta, como si Alba hubiera experimentado con una orientación sexual "provisional" antes de descubrir la "verdadera".

\subsection{Sus percepciones, experiencias y actitudes frente a las desigualdades vinculadas al género}

Merece la pena prestar atención a las percepciones de estas chicas y estos chicos, en principio igualitarios y respetuosos con la diversidad, acerca de las desigualdades vinculadas al género; tanto las asociadas a las identidades normativas, como las que experimentan quienes se apartan de la heteronormatividad; ya que la mayor parte de ellas les pasan desapercibidas y en bastantes ocasiones muestran incongruencias entre sus discursos y sus actitudes.

En relación a las desigualdades propias de las identidades normativas, debemos destacar que solo las chicas detectan algunas, mientras que son ignoradas por los chicos.

Alicia, lejos de señalar la subordinación femenina, destacaba como generadoras de desigualdad algunas conductas de riesgo que autores como Lomas (2004) o Hernández, Videla, Herráiz y Sancho (2007) consideran típicamente masculinas, las cuales observaba en el grupo de amigos de su primer novio; como la agresividad y el consumo de drogas. Estas, desde su punto de vista, habían contribuido a generar en el chico un carácter callado e inmaduro.

Por su parte, Elena identificaba injusticias en las relaciones amorosas de sus compañeras de instituto y de sus propias amigas. La chica las consideraba totalmente subordinadas a sus parejas, especialmente en el ámbito de la sexualidad, ya estas no se responsabilizaban de contar con preservativos y estaban dispuestas a asumir embarazos no deseados por complacer los deseos sexuales de sus novios; en la línea de los hallazgos de Navarro, Barberá, Reig y Ferrer (2006). Por otra parte, criticaba las normas de vestuario discriminatorias que establecen los pubs a las chicas.

Pero para Fernando y David las desigualdades entre chicas y chicos pasan desapercibidas. El primero afirmaba con 15 años que en su pandilla nadie actuaba según marcan los estereotipos de género, que todos eran "neutros". Si bien posteriormente explicaba que los chicos seguían manteniendo actitudes machistas como molestarse porque sus novias salieran con sus amigas (a pesar de que ellos hacían lo mismo con sus amigos), o no contar con ellas para tomar decisiones. Con 17 expone que en nuestra sociedad ya no se reproducen los estereotipos de género y lo ejemplifica haciendo referencia a los chicos que se depilan, como si este acto contribuyera a la igualdad. 
David tampoco detecta desigualdades entre chicos y chicas, y sólo tras algo de insistencia reconoce que la mayoría de sus amigas deben volver más temprano a casa que sus amigos. El chico asegura que los adolescentes no son machistas, no identifica el sexismo en el reggaetón ni en las series manga que tanto le gustan y justifica los comentarios despectivos que usualmente reciben las chicas que salen con muchos chicos, argumentando que son bromas y que se dirigen igualmente a los chicos. Tampoco percibe diferencias entre géneros en lo que se refiere a las conductas violentas, ni en los aspectos académicos. En lo relativo a las labores domésticas, indica que él hace más que su hermana. También piensa que los chicos cercanos a él no se sienten forzados a ocultar sus sentimientos, a ser violentos y, en general, a representar los estereotipos de género; no obstante, es consciente de que en muchos casos, a los niños se les califica de "mariquitas" cuando lloran.

Al hilo de los insultos homófobos, son destacables algunos comentarios que nuestros protagonistas dirigen a ciertos grupos de jóvenes que escapan del binarismo, resultando especialmente curiosos los que parten de Fernando y de Elena, al apartarse ellos mismos de la heterosexualidad. Para facilitar la comprensión de esta compleja cuestión, es pertinente relatar previamente las vivencias difíciles asociadas a la homosexualidad que ambos han experimentado.

Atendiendo a los comentarios de Fernando, parece que nunca se ha sentido mal por esa causa ni rechazado por otras personas. No obstante, ha tenido que afrontar determinadas situaciones puntuales con alguna dificultad añadida. Por ejemplo, al hablarnos acerca de sus preferencias sexuales, para introducir su exposición, intenta reflejar la confusión que ha experimentado al respecto afirmando que está "liado". Además, añade que de más pequeño le resultaba más complicada la situación y que tuvo que concienciarse a sí mismo de que no había ningún problema por el hecho de que se sintiera atraído por chicos. En la misma línea, Fernando ha evitado mantener conversaciones relativas a sus opciones sexuales con sus padres, aunque sospechara que estos estaban al corriente de ellas y las aceptaban.

Para Elena la situación ha sido bastante más complicada, la homofobia ha condicionado su vida desde que con 8 años expusiera en clase que a ella le gustaban las mujeres. A partir de ese momento comenzó a volverse agresiva para defenderse de las burlas de sus compañeras, lo que la llevó a las consultas de diversos psicólogos que no pudieron ayudarla, debido a que la actitud de negación de su homosexualidad por parte de su madre motivó que no explicara a estos el origen de los conflictos. Es destacable el papel de la madre de Elena, quien doce años después de conocer la orientación homosexual de su hija sigue sin aceptarla, lo que la lleva a interpelarla continuamente para que desarrolle el papel normativo y salga con chicos, escudándose en el argumento de que quiere facilitarle la vida.

Del mismo modo, la homofobia dominante ha estado limitando la libertad de Elena en el desarrollo de sus relaciones amorosas, ya que el temor de sus últimas parejas a las reacciones de sus padres ha dado lugar a que durante toda su adolescencia y juventud haya tenido que mantenerlas a escondidas.

Una vez conocidas las circunstancias de estos dos adolescentes, la diversidad de percepciones y actitudes hacia quienes no asumen identidades normativas resultará más comprensible e interesante. 
Por ejemplo, David, que ha tenido un contacto bastante superficial con la homosexualidad, exclusivamente a través de algunas amistades a las que considera "muy sueltas", no es consciente de las complicaciones que esta conlleva, y piensa que dichas amistades no se han encontrado con muchas más dificultades que otros chicos y chicas heterosexuales.

Por su parte, Elena, que ha experimentando la homofobia toda su vida y no entiende que en nuestros días haya personas que la expresen de manera tan explícita como lo han hecho los padres de algunas de sus novias, muestra su rechazo hacia chicas de su pueblo que, en su opinión, son demasiado jóvenes para ir manifestando públicamente su lesbianismo y solo lo hacen por estar esa práctica de moda en su localidad. Parece que considerara que estas chicas no se toman en serio algo que tanto sufrimiento le ha generado a ella. Sin embargo, por otra parte, le encanta que, de los cuatros miembros que componen el consejo local de la juventud de su pueblo, tres sean homosexuales, de modo que esta identidad se visibilice.

Alicia, que no descarta mantener relaciones homosexuales por el simple hecho de experimentar, reconoce que le costaría definirse públicamente como lesbiana, ya que es consciente de las complicaciones que ello le generaría; por ejemplo, en el vestuario con sus compañeras de equipo. No obstante, a pesar de sus críticas hacia la homofobia, alude en tono jocoso a las chicas de un equipo de futbol femenino de su pueblo, las cuales son objeto de las bromas de sus compañeras de baloncesto por definirse en su mayoría como lesbianas. Estas bromas probablemente estarían relacionadas con la idea que expone Fernando sobre que "las chicas suelen apoyar a los chicos homosexuales y los chicos suelen apoyar a las chicas homosexuales", la cual ejemplificaba aludiendo, en voz baja y con cierto tono despectivo, a una compañera de su clase "que es así (lesbiana)" y tiene como amigos a otros compañeros suyos que defienden que la homosexualidad es una enfermedad. El chico quedó totalmente asombrado al descubrir que sigue habiendo jóvenes que entienden que ser gay es "como ser autista". Sin embargo, esta explicación es similar a la que Elena ofrecía a su madre con intención de que esta la aceptara, comparando su homosexualidad con ser "subnormal".

Pero, a pesar de su diferente comprensión de la homosexualidad, Fernando y Elena, comparten su rechazo a identificarse con quienes alardean de ella, como en el Día del Orgullo Gay; quizás porque lo que persiguen es sentirse adaptados y aceptados en el sistema heteronormativo. Al contrario que Alicia, a quien su comprensión del carácter performativo del proceso de construcción de la identidad le anima a trasgredir las normas de género, que reconoce como acuerdos sociales, y la lleva manifestar que si se identificara como bisexual participaría en el citado desfile.

\section{Nuestros PRinCiPAles hallazgos}

Como adelantábamos, el primer aspecto destacable de nuestro trabajo lo encontramos en que, en solo cuatro casos, refleja la diversidad existente entre los chicos y las chicas adolescentes; aspecto que consideramos de gran valor, ya que lo habitual es la difusión de modelos estereotipados, y en su defecto, modelos en situación de exclusión. 
El segundo aspecto que consideramos de interés se refiere a la gran importancia que conceden estos y estas jóvenes a la influencia de sus padres y madres en la construcción de sus propias identidades, lo que nos proporciona pistas acerca de hacia dónde dirigir la intervención educativa.

Otro aspecto de gran importancia se refiere a la ausencia de conocimientos acerca de cómo se construyen las identidades de género. Resulta significativo que, al entender Alicia que las identidades se construyen de manera performativa (representando los modelos que nuestra sociedad nos ofrece para las categorías en las que nos ha ubicado), sea capaz de cuestionarse dichas categorías de género y, en consecuencia, se sienta más libre para transgredirlas. Esto nos incita a pensar que la difusión de esta perspectiva teórica podría contribuir a reducir la presión social para el cumplimiento de las normas de género que se nos imponen.

Relevante igualmente es el hecho de que, para estos adolescentes, las desigualdades existentes entre los dos género normativos pasen casi desapercibidas. No obstante, las detectadas por Alicia y por Elena merecen nuestra atención, ya que, tanto las conductas de riesgo y la violencia típicamente masculina, como la subordinación femenina en las relaciones sexuales, son generadoras de gran cantidad de situaciones de desigualdad.

En último lugar, debemos mencionar el rechazo y la burla que generan determinados modelos identitarios no normativos entre estos chicos y chicas (supuestamente) sensibilizados hacia las desigualdades vinculadas a la diversidad de género. Estas actitudes son muestra de la profunda interiorización de determinados prejuicios, que en ocasiones surgen con más fuerza y espontaneidad que las ideas igualitarias razonadas y reflexionadas.

\section{LAS PROPUESTAS EDUCATIVAS QUE SURGEN DE NUESTRO TRABAJO}

El panorama que dibujan nuestros casos nos invita a pensar que la labor educativa debe abarcar desde la aclaración de los conceptos básicos relacionadas con las identidades de género, hasta la comprensión y la valoración de la riqueza de la diversidad humana. Más específicamente, y siempre a la luz de los conocimientos adquiridos junto a nuestros protagonistas, destacamos las siguientes necesidades de intervención educativa.

En primer lugar, vemos la necesidad de extender la formación y la sensibilización en relación a la diversidad de género a las familias del alumnado, debido a la influencia determinante que tienen sobre este, al constituir su primer agente de socialización.

La segunda propuesta se orienta al acercamiento de la perspectiva performativa de la construcción de la identidad a la escuela. Es importante que desde los niveles iniciales del sistema educativo no se aborde la cuestión del binarismo de género como si esta clasificación fuera una realidad natural y, por tanto, que no se imponga al alumnado identificarse como niño o como niña; que se adopte una actitud inclusiva, evitando tratarlos en función de las etiquetas de género que se les han asignado, sino atendiendo a sus características y necesidades particulares, favoreciendo la diversidad y la libertad. 
Nuestra tercera propuesta, íntimamente ligada a la anterior, se refiere a la difusión de modelos de identidad no estereotipados, más diversos e igualitarios; a partir de cuentos, películas, libros de textos, etc.; que constituyan nuevos referentes con los que los niños y las niñas puedan identificarse.

Además de favorecer el desarrollo de identidades diversas, igualitarias y felices, es necesario hacer consciente al alumnado de que la sociedad no siempre nos facilita este proceso y de que en muchos casos la clasificación de las personas en función del sexo, el género y la orientación sexual da lugar a desigualdades. Es nuestra responsabilidad hacerles visibles los actos que implican sexismo, homofobia, transfobia o cualquier tipo de discriminación vinculada a la división binaria de los géneros, para que puedan posicionarse frente a ellas. La última propuesta educativa apunta a la educación emocional y sexual para combatir la violencia y la subordinación.

Obviamente, para que la educación aborde este tipo de cuestiones, sus principales responsables, los maestros y las maestras, deben estar sensibilizados y contar con la formación necesaria; y deben ofrecer a su alumnado experiencias de reflexión, de crítica, de responsabilidad, de libertad... Solo de esta manera podrá entender que es posible cambiar todo lo que ha sido construido socialmente y que es posible un mundo más igualitario y justo.

\section{REFERENCIAS BIBLIOGRÁFICAS}

Alonso, I. (22-08-2013). Las organizaciones de intersexuales rechazan el 'tercer género' que propone Alemania. Huffingtonpost. Recuperado de: http://www. huffingtonpost.es/2013/08/22/intersexual-genero-alemania_n_3793897.html

American Psychiatric Association (2014). DSM-5 Manual diagnóstico y estadístico de los trastornos mentales. Buenos Aires: Panamericana.

Ayuso, B. (17-09-2016). Soy intersexual, no hermafrodita. El País. Recuperado de: http://elpais.com/elpais/2016/09/17/actualidad/1474075855_705641.html

Bolin, A. (2003). La transexualidad del género. Contexto cultural y prácticas de género. En J. A. Nieto, Antropología de la sexualidad (pp. 231-259). Madrid: Talasa.

Butler, J. (2007). El género en disputa. El feminismo y la subversión de la identidad. Barcelona: Paidos.

Butler, J. (2008). Cuerpos que importan. Buenos Aires: Paidos.

Duque, E. (2006). Aprendiendo para el amor o para la violencia. Las relaciones en las discotecas. Barcelona: El Roure.

Fausto-Sterling, A. (1998). Los cinco sexos. ¿Por qué varón y mujer no son suficientes? En J. A. Nieto, (Comp.), Transexualidad, transgenerismo y cultura. Antropología, identidad y género (pp. 79-89). Madrid: Talasa.

Guasch, O. (2007). La crisis de la heterosexualida. Barcelona: Laertes.

Generelo, J. y Pichardo, J. I. (Coord.) (2006). Homofobia en el sistema educativo. Madrid: COGAM.

Hernández, F., Vidiella, J., Herraiz, F. y Sancho, J. M. (2007). El papel de la violencia en el aprendizaje de las masculinidades. Revista de educación, 342, 103-125. 
Ley 2/2014 de 8 de julio, integral para la no discriminación por motivos de identidad de género y reconocimiento de los derechos de las personas transexuales de Andalucía. BOJA núm. 139, 18 de julio.

Lomas, C. (Comp.) (2004). Los chicos también Iloran. Identidades masculinas, igualdad entre los sexos y coeducación. Barcelona: Paidos.

Moreno, A. (1988). La otra política de Aristóteles: cultura de masas y divulgación del arquetipo viril. Barcelona: Icaria.

Navarro Pertusa, E., Reig Ferrer, A., Barberá Heredia, E. y Ferrer Cascale, R. (2006). Grupos de iguales e iniciación sexual adolescente: diferencias de género. International Journal of Clinical and Health Psicology, 6(1), 79-96.

Penna Tosso, M. (2015). Homofobia en las aulas universitarias. Un meta-análisis. Revista de docencia universitaria, 13(1), 181-202.

Penna Tosso, M. y Sánchez Sáinz, M. (2015). Evaluación de la homofobia en los futuros docentes de Educación Secundaria. Revista de Investigación Educativa, 33(1), 83-98.

Pérez Gómez, A. I. (1992). Las funciones sociales de la Escuela. En J. Gimeno Sacristán y A. I. Pérez Gómez, Comprender y transformar la enseñanza (pp. 17 33). Madrid: Morata.

Pichardo Galán, J. I. (2009). Adolescentes ante la diversidad sexual. Madrid: Catarata. Platero, R. (2008). La homofobia como elemento clave del acoso escolar homofóbico. Algunas voces desde Rivas Vaciamadrid. Información psicológica 94, 71-83.

Platero, R. (2010). Sobrevivir al Instituto y a la Facultad: voces y vivencias sobre la heteronormatividad, la homofobia y la masculinidad de las chicas. Revista de estudios de juventud. Discriminaciones diversas en las personas jóvenes, 89, 39-58.

Renold, E. (2000). Coming out gender, (hetero)sexuality and the primary school. Gender and education, 12(3), 309-326.

Renold, E. (2001a). Learning the "hard" way: boys, hegemonic masculinity and the negotiation of learner identities in the primary school. British journal of sociology of education, 22(3), 386-395.

Renold, E. (2001b). Square-girls, feminity and the negotiation of academic success in the primary school. British educational research journal, 27(5), 577-588.

Renold, E. (2003). If you don't kiss me you are dumped: boys, boyfriends and heterosexualised masculinities in the primary school. Educational review, 55(2), 179-194.

Renold, E. (2004). Other boys: negotiation non hegemonic masculinities in the primary school. Gender and education, 16(2), 247-266.

Rubin, G. (1996). El tráfico de mujeres: Notas sobre la "economía política" del sexo. En Marta Lamas (Comp.), El género: la construcción cultural de la diferencia sexual (pp. 35-96). México: PUEG (1ª edición de 1975).

Sainz Sánchez, M. (2014). Género, diversidades y diferencia en Educación Infantil y Primaria. Madrid: Fundación $1^{\circ}$ de Mayo.

Simons, H. (2011). El estudio de caso: teoría y práctica. Madrid: Morata.

Vázquez García, F. (2007). Del hermafroditismo al transexual. Elementos para una genealogía del cuerpo sexuado. En N. Corral (Coord.), Prosa corporal (pp. 75-97). Madrid: Talasa Ediciones. 
Vázquez García, F. (2009). Políticas transgénicas y ciencias sociales: por un construccionismo bien temperado. En Ponencias Seminario: Teoría Queer. De la transgresión a la transformación social (pp. 3-14). Sevilla: Centro de Estudios Andaluces.

Vidarte, F. J., Saez, J. y Córdoba, D. (Eds.) (2007). Teoría Queer. Políticas bolleras, maricas, trans, mestizas. Barcelona: Egales.

Wolcott, H. F. (1994). Transforming Qualitative Data: Description. Analysis and Interpretation. Londres: Sage. 